University of New Hampshire

University of New Hampshire Scholars' Repository

$6-2004$

\title{
Evidence for partial quenching of orbital angular momentum upon complex formation in the infrared spectrum of $\mathrm{OH}$-acetylene
}

\author{
Mark D. Marshall \\ James B. Davey \\ Margaret E. Greenslade \\ University of New Hampshire, Margaret.E.Greenslade@unh.edu \\ Marsha I. Lester
}

Follow this and additional works at: https://scholars.unh.edu/chemistry_facpub

Part of the Physical Chemistry Commons

\section{Recommended Citation}

M. D. Marshall, J. B. Davey, M. E. Greenslade, M. I. Lester, “Evidence for partial quenching of orbital angular momentum upon complex formation in the infrared spectrum of $\mathrm{OH}$-acetylene," J. Chem. Phys. 121,5845 (2004).

This Article is brought to you for free and open access by the Chemistry at University of New Hampshire Scholars' Repository. It has been accepted for inclusion in Chemistry Scholarship by an authorized administrator of University of New Hampshire Scholars' Repository. For more information, please contact Scholarly.Communication@unh.edu. 


\section{AIP $\begin{gathered}\text { mesoumalot } \\ \text { chemical Physics }\end{gathered}$}

\section{Evidence for partial quenching of orbital angular momentum upon complex formation in the infrared spectrum of $\mathrm{OH}$-acetylene}

Mark D. Marshall, James B. Davey, Margaret E. Greenslade, and Marsha I. Lester

Citation: J. Chem. Phys. 121, 5845 (2004); doi: 10.1063/1.1784444

View online: http://dx.doi.org/10.1063/1.1784444

View Table of Contents: http://jcp.aip.org/resource/1/JCPSA6/v121/i12

Published by the American Institute of Physics.

\section{Additional information on J. Chem. Phys.}

Journal Homepage: http://jcp.aip.org/

Journal Information: http://jcp.aip.org/about/about_the_journal

Top downloads: http://jcp.aip.org/features/most_downloaded

Information for Authors: http://jcp.aip.org/authors

\section{ADVERTISEMENT}
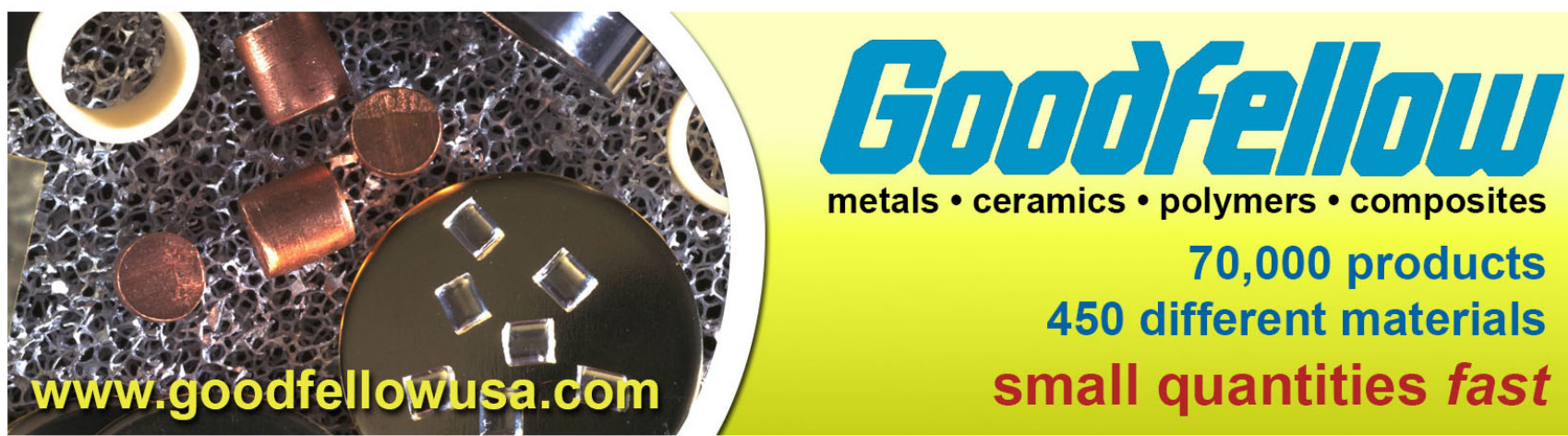

metals • ceramics $\bullet$ polymers $\bullet$ composites

70,000 products 450 different materials small quantities fast 


\title{
Evidence for partial quenching of orbital angular momentum upon complex formation in the infrared spectrum of $\mathrm{OH}$-acetylene
}

\author{
Mark D. Marshalla) \\ Department of Chemistry, Amherst College, Amherst, Massachusetts 01002-5000 \\ James B. Davey, ${ }^{\text {b) }}$ Margaret E. Greenslade, and Marsha I. Lester ${ }^{\text {c) }}$ \\ Department of Chemistry, University of Pennsylvania, Philadelphia, Pennsylvania 19104-6323
}

(Received 20 May 2004; accepted 28 June 2004)

\begin{abstract}
The entrance channel leading to the addition reaction between the hydroxyl radical and acetylene has been examined by spectroscopic characterization of the asymmetric $\mathrm{CH}$ stretching band of the $\pi$-hydrogen bonded $\mathrm{OH}$-acetylene reactant complex. The infrared action spectrum observed at $3278.6 \mathrm{~cm}^{-1}$ (origin) consists of seven peaks of various intensities and widths, and is very different from those previously reported for closed-shell $\mathrm{HF} / \mathrm{HCl}$-acetylene complexes. The unusual spectrum arises from a partial quenching of the $\mathrm{OH}$ orbital angular momentum in the complex, which in turn is caused by a significant splitting of the $\mathrm{OH}$ monomer orbital degeneracy into ${ }^{2} A^{\prime}$ and ${ }^{2} A^{\prime \prime}$ electronic states. The magnitude of the ${ }^{2} A^{\prime}-{ }^{2} A^{\prime \prime}$ splitting as well as the $A$ rotational constant for the $\mathrm{OH}$-acetylene complex are determined from the analysis of this $b$-type infrared band. The most populated $\mathrm{OH}$ product rotational state, $j_{\mathrm{OH}}=9 / 2$, is consistent with intramolecular vibrational energy transfer to the $\nu_{2} \mathrm{C} \equiv \mathrm{C}$ stretching mode of the departing acetylene fragment. The lifting of the $\mathrm{OH}$ orbital degeneracy and partial quenching of its electronic orbital angular momentum indicate that the electronic changes accompanying the evolution of reactants into products have begun to occur in the reactant complex. (C) 2004 American Institute of Physics. [DOI: 10.1063/1.1784444]
\end{abstract}

\section{INTRODUCTION}

Reactions of open-shell diatomic radicals with closedshell partners raise important, interesting questions regarding the influence of electronic orbital angular momentum, present in the radical reactant (if not in a $\Sigma$ state), as the system evolves along the reaction coordinate to products in which this momentum is quenched. For the hydroxyl radical $(\mathrm{OH})$, which is of particular relevance in atmospheric ${ }^{1}$ and combustion $^{2}$ processes, the orbital degeneracy of the ${ }^{2} \Pi$ ground state is resolved into separated ${ }^{2} A^{\prime}$ and ${ }^{2} A^{\prime \prime}$ electronic states upon approach of the closed-shell partner in nonlinear geometries. Typically only one of these two electronic states leads to reaction, ${ }^{3}$ but as long as the energy difference between the two is smaller than the $140 \mathrm{~cm}^{-1}$ spin-orbit splitting of the $\mathrm{OH}$ radical the states remain mixed and the electronic orbital angular momentum is not significantly quenched.

We have recently characterized a $T$-shaped, $\pi$-type hydrogen bonded reactant complex between the hydroxyl radical and acetylene $\left(\mathrm{C}_{2} \mathrm{H}_{2}\right),{ }^{4}$ which may play a role in the electrophilic addition of $\mathrm{OH}$ to the $\pi$-bond of acetylene, the dominant mechanism for reaction between the two species near room temperature. The complex was determined to have the $\mathrm{H}$ atom of $\mathrm{OH}$ pointed toward the acetylene, as shown in the inset to Fig. 1, with the $\mathrm{O}$ atom $3.385 \AA$ away from

a) On sabbatical leave at the University of Pennsylvania during 2003-04.

${ }^{b)}$ Present address: Department of Chemistry, University of Leeds, Leeds, UK LS2 9JT.

${ }^{c)}$ Author to whom all correspondence should be addressed; Fax (215) 573-

2112; Electronic mail: milester@ sas.upenn.edu center of the $\mathrm{C} \equiv \mathrm{C}$ bond. Also shown in Fig. 1 are the calculated energies of the ${ }^{2} A^{\prime}$ and ${ }^{2} A^{\prime \prime}$ states as a function of the separation of the partners in a $T$-shaped arrangement and the difference potential, $\rho=E\left({ }^{2} A^{\prime \prime}\right)-E\left({ }^{2} A^{\prime}\right)$. As detailed in previous work, ${ }^{4}$ the $a b$ initio calculations were carried out at the restricted coupled cluster singles, doubles, noniterative triples RCCSD(T) level of theory with extrapolation to the complete basis set limit. At all distances for this geometry, the ${ }^{2} A^{\prime \prime}$ state, in which the unpaired electron occupies an O-centered, $p \pi$ orbital perpendicular to the plane of the complex, is predicted to be lower in energy than the ${ }^{2} A^{\prime}$ state, which has the unpaired electron in an analogous inplane $p \pi$ orbital. For other orientations of the two reactive partners, the $a b$ initio results have the ${ }^{2} A^{\prime}$ state as the lower of the two, and it is this state that leads to reaction and the radical, intermediate product, $\mathrm{HCCHOH}$. Thus, in addition to the familiar changes in bond lengths, bond angles, and atomic connectivity as the $\mathrm{OH}+\mathrm{C}_{2} \mathrm{H}_{2}$ system proceeds along the reaction coordinate from reactants to products, the difference potential also changes, starting at zero when the species are far apart and increasing in magnitude upon approach to the transition state. Quenching of electronic orbital angular momentum occurs as the magnitude of the difference potential increases, and the electronic description of the system evolves from a spin-orbit coupled ${ }^{2} A^{\prime}-{ }^{2} A^{\prime \prime}$ mixed state to (in this case) the ${ }^{2} A^{\prime}$ ground state of $\mathrm{HCCHOH}$. Characterization of the $\mathrm{OH}$-acetylene reactant complex provides a snapshot of the system in the intermediate region.

The previous study of the $\mathrm{OH}$-overtone stretching band in OH-acetylene demonstrated that the complex still possesses electronic orbital angular momentum, but could not 


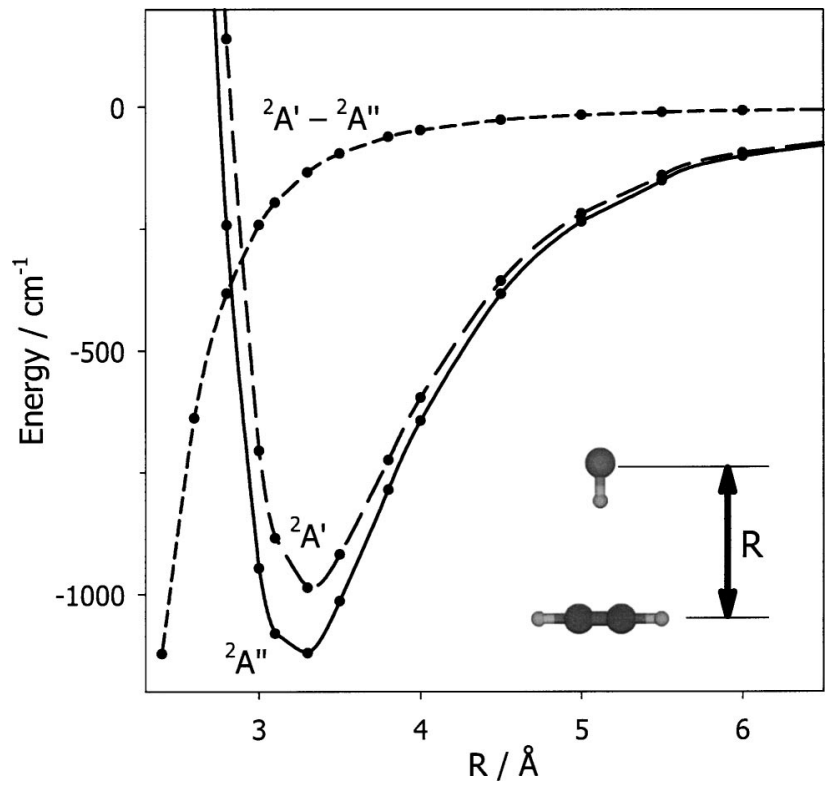

FIG. 1. Radial cuts (smoothed curves) of the $\mathrm{OH}+\mathrm{C}_{2} \mathrm{H}_{2}$ intermolecular potential surface for a fixed, $T$-shaped orientation of the monomers with the $\mathrm{H}$ atom of $\mathrm{OH}$ directed towards the $\pi$-bond of acetylene as shown in the inset. The ${ }^{2} A^{\prime}$ and ${ }^{2} A$ " curves (long dash and solid lines) correspond to the half-filled $p \pi$ orbital of the $\mathrm{OH}$ radical lying within or perpendicular to the plane of the complex, respectively. The short dash line shows the radial dependence of the difference potential, $\rho=E\left({ }^{2} A^{\prime}\right)-E\left({ }^{2} A^{\prime \prime}\right)$, which is zero at large monomer separation and becomes increasingly negative as the monomers approach each other in this orientation. The difference potential is responsible for the quenching of electronic orbital angular momentum. The $a b$ initio potentials are adapted from Fig. 2 of Ref. 4, where calculations were carried at the $\operatorname{RCCSD}(\mathrm{T})$ level of theory with extrapolation to the complete basis set limit.

provide a definite conclusion regarding the degree of departure from the completely unquenched limit. ${ }^{4}$ A model developed to calculate the rotational energy levels and electric dipole transition intensities in $\mathrm{OH}$-containing complexes with intermediate values of the difference potential suggested that the $b$-type, asymmetric $\mathrm{CH}$ stretching band of the complex would be more sensitive to the effects of orbital angular momentum quenching. ${ }^{5}$ We present here the infrared action spectrum and analysis of this band, which clearly show that the $\mathrm{OH}$-acetylene reactant complex has a difference potential comparable in magnitude to the $\mathrm{OH}$ spinorbit splitting, and conforms to neither limiting case of completely mixed nor well-separated ${ }^{2} A^{\prime}$ and ${ }^{2} A$ " electronic states.

Structural comparisons with analogous closed-shell complexes indicate that similar interactions are responsible for complex formation in those species and $\mathrm{OH}$-acetylene at this point along the $\mathrm{OH}+\mathrm{C}_{2} \mathrm{H}_{2}$ reaction coordinate. However, the significant effects on the rotational energy levels and infrared band contour of $\mathrm{OH}$-acetylene signal the start of the changes in the electronic environment of the species. These changes include the partial quenching of the $\mathrm{OH}$ orbital angular momentum, a process that must occur as the reactive partners approach the transition state to reaction.

\section{EXPERIMENT}

Binary complexes composed of the $\mathrm{OH}$ and $\mathrm{C}_{2} \mathrm{H}_{2}$ reactants are produced by photolyzing $\mathrm{HNO}_{3}$ entrained in a gas mixture of $10 \% \mathrm{C}_{2} \mathrm{H}_{2}$ in $\mathrm{Ar}$ at $60 \mathrm{psi}$ at the initial stage of a pulsed supersonic expansion, as described previously. ${ }^{4} \mathrm{Pho}-$ tolysis takes place near the exit of a quartz capillary tube $(0.3$ $\mathrm{mm}$ ID) affixed to the pulsed valve. The infrared action spectrum of the $\mathrm{OH}$-acetylene reactant complex is recorded using an IR pump-UV probe technique. ${ }^{4,6}$ The IR pump laser excites the $\mathrm{OH}$-acetylene complex in the asymmetric acetylenic $\mathrm{CH}$ stretching region, and the UV probe laser detects $\mathrm{OH}$ $(v=0)$ fragments by saturated laser-induced fluorescence in the $\mathrm{OH} A^{2} \Sigma^{+}-X^{2} \Pi(1,0)$ region.

Tunable infrared radiation at $\sim 3.0 \mu \mathrm{m}$ is generated by an optical parametric oscillator (OPO) (Laservision, 0.15 $\mathrm{cm}^{-1}$ linewidth) pumped by an injection-seeded Nd:yttrium aluminum garnet (YAG) laser (Continuum Precision II 8000; $8 \mathrm{~ns}$ pulse, $10 \mathrm{~Hz}$ repetition rate). The OPO delivers up to 10 $\mathrm{mJ} /$ pulse of IR radiation in this spectral region. The absolute frequency of the OPO is determined by recording an $\mathrm{NH}_{3}$ photoacoustic spectrum as the infrared laser is scanned, and comparing to well-documented transitions in the HITRAN database. $^{7}$

The UV probe beam is generated by the frequency doubled output of a Nd:YAG pumped dye laser $(20 \mathrm{~Hz})$ operating with R6G dye, which produces tunable radiation around $282 \mathrm{~nm}$. Typically, $\sim 1 \mathrm{~mJ} /$ pulse of UV radiation is passed into the vacuum chamber. The UV laser is calibrated using the well-known frequencies of the $\mathrm{OH} A-X(1,0)$ lines. ${ }^{8}$

The IR and UV laser beams are counterpropagated, focused, and spatially overlapped in the vacuum chamber $\approx 15$ $\mathrm{mm}$ downstream from the exit of the capillary tube. The focused beams measure $\approx 1 \mathrm{~mm}^{2}$ in the interaction region. Several filters and a photomultiplier gating circuit were used to block scattered light arising from the photolysis and UV probe lasers, while still passing fluorescence in the $\mathrm{OH}$ $A-X(1,1)$ spectral region. An active background subtraction technique is used in processing the data in order to subtract the background signal observed with the UV laser alone (20 $\mathrm{Hz}$ ) from the infrared-induced signal obtained with both IR and UV lasers present $(10 \mathrm{~Hz})$. The background signal arises from uncomplexed $\mathrm{OH}$ in the supersonic expansion. The $\mathrm{OH}$ $A-X(1,0) Q_{1}(1)$ line could not be probed due to large background from $\mathrm{OH}$ cooled to its lowest rovibrational state, and $Q_{1}(2)$ could not be probed due to overlap with an $\mathrm{OH}-\mathrm{Ar}$ feature. ${ }^{9}$

\section{RESULTS AND ANALYSIS}

A broad spectroscopic feature spanning 3273-3286 $\mathrm{cm}^{-1}$ and requiring the presence of both photolytically generated $\mathrm{OH}$ radicals and acetylene in the molecular jet was observed in the region corresponding to the asymmetric $\mathrm{CH}$ stretching band $\left(\nu_{3}\right)$ of acetylene. Although this vibration is part of a Fermi dyad in the acetylene monomer, this resonance with $\nu_{2}+\nu_{4}+\nu_{5}$ of the monomer is quenched in all previously studied complexes of acetylene ${ }^{10-14}$ with the exception of those with rare gases ${ }^{15-17}$ and $\mathrm{CO}_{2}$-acetylene. ${ }^{18}$ The observed band is shown in Fig. 2; several scans were averaged to obtain the figure. This infrared action spectrum was recorded with the UV probe laser fixed on the $\mathrm{OH}$ 


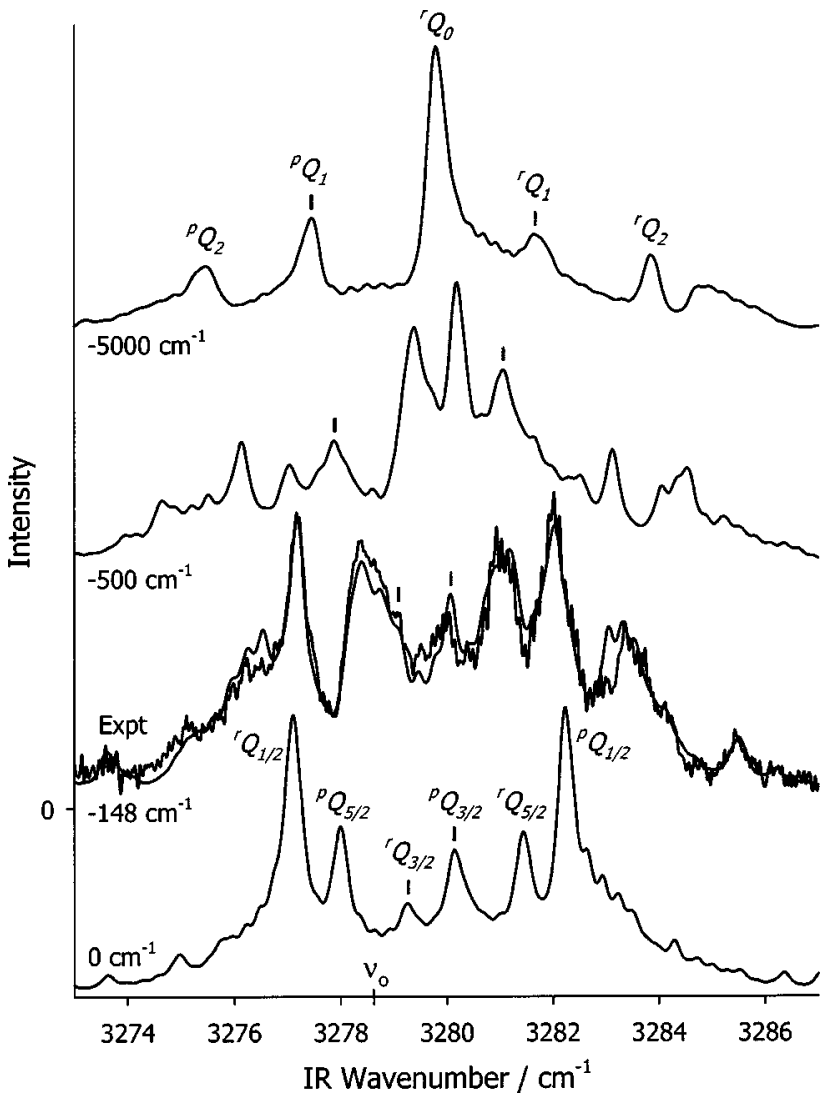

FIG. 2. Simulations of the asymmetric $\mathrm{CH}$ stretching fundamental band for four different values of the difference potential $\rho$ showing its effect on the band contour. The upper and lower traces, corresponding to completely quenched and completely unquenched electronic orbital angular momentum, respectively, are dominated by $Q$ branches (see text for a description of the labeling). The evolution of the ${ }^{p} Q_{3 / 2}\left({ }^{r} Q_{3 / 2}\right)$ feature in the bottom spectrum through the intermediate cases into the ${ }^{p} Q_{1}\left({ }^{r} Q_{1}\right)$ of the top is shown by the black (gray) bars. The simulation at $\rho=-148 \mathrm{~cm}^{-1}$ is overlaid with the experimental spectrum, demonstrating the excellent agreement between the two.

$A-X(1,0) Q_{1}(4)$ transition, which was empirically found to give the strongest infrared induced signal and the best signal to background ratio. As evident from the figure, the band structure is complicated and is not immediately recognizable as a perpendicular, $b$-type infrared spectrum arising from the $T$-shaped $\mathrm{OH}$-acetylene complex.

Given that the previously analyzed $\mathrm{OH}$ overtone band of the complex indicates that the orbital angular momentum of the hydroxyl radical remains largely unquenched, ${ }^{4}$ it is not surprising that the present band differs from that of covalently bound radical with a ${ }^{2} A$ " electronic ground state. For such a molecule, the band would have the form shown in the top trace of Fig. 2 and be qualitatively similar to the $b$-type bands observed previously, albeit at much higher resolution, for the asymmetric $\mathrm{CH}$ stretches of the $T$-shaped, closed-shell complexes of acetylene with $\mathrm{HF},{ }^{10} \mathrm{HCl},{ }^{11}$ and HCN. ${ }^{14}$ As can be seen by comparison with the bottom trace in Fig. 2, however, neither does this band in $\mathrm{OH}$-acetylene have the Coriolis-split $Q$-branch pattern that would be expected if the electronic orbital angular momentum were completely unquenched. In this limit, the spectrum would be similar to that of a symmetric top molecule in a degenerate

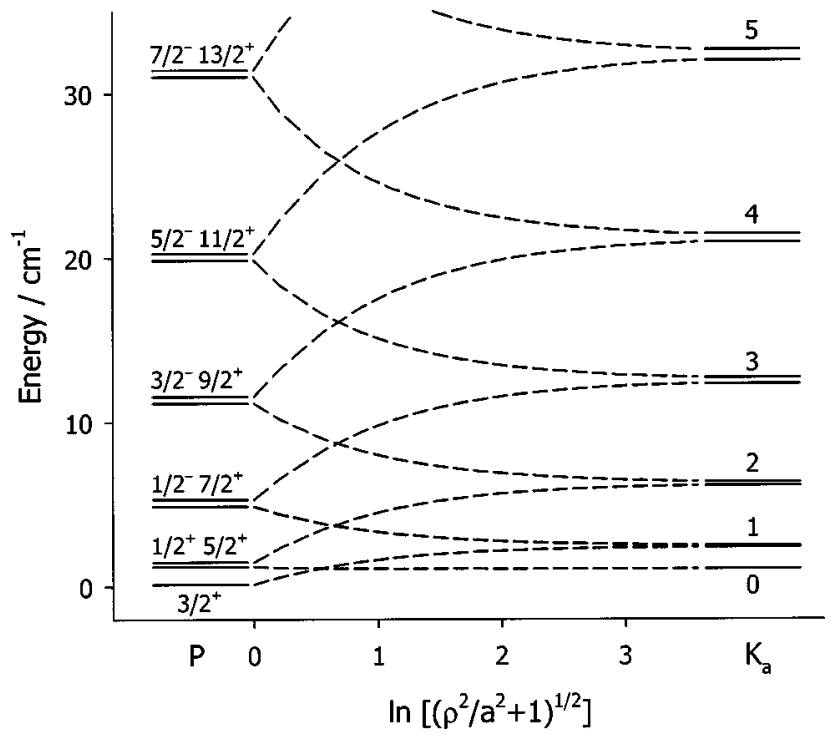

FIG. 3. Correlation diagram showing the energies (minus $\sqrt{\rho^{2}+a^{2}} / 2$ ) of the rotational manifolds for $\mathrm{OH}$-acetylene as a function of the ratio of the difference potential $\rho$ to the monomer spin-orbit coupling constant $a$. The levels on the left are labeled with the value of the half integer quantum number $P$ and a superscript indicating the sign of $\omega$. The left (right) hand label refers to the lower (upper) half of each pair of levels, and $|\omega|=3 / 2$ for all the levels shown. As the difference potential increases, the electronic orbital angular momentum is quenched and the manifolds eventually rearrange into the limiting case on the right in which levels are characterized by the familiar quantum number $K_{a}$.

electronic state such as the methoxy radical. ${ }^{19}$ Indeed, as will be discussed more fully below, the band contour clearly shows that $\mathrm{OH}$-acetylene is an intermediate case, providing an example of a molecule in which orbital angular momentum is partially quenched, a process that must occur along the reaction coordinate from $\mathrm{OH}+\mathrm{C}_{2} \mathrm{H}_{2}$ to the radical intermediate, $\mathrm{HCCHOH}$.

Marshall and Lester have developed a model to treat the rotational energy level structure of $\mathrm{OH}$-containing complexes with arbitrary quenching of the electronic orbital angular momentum and to predict their infrared spectra. ${ }^{5}$ The splitting of the $\mathrm{OH}$ monomer orbital degeneracy into ${ }^{2} A^{\prime}$ and ${ }^{2} A^{\prime \prime}$ electronic states that is responsible for the quenching is incorporated directly into the model Hamiltonian via the operator,

$$
\hat{H}_{q}=\frac{\rho}{2}\left(\bar{l}_{+}^{2}+\bar{l}_{-}^{2}\right),
$$

where $\rho$ is the difference potential, and the bars over the electronic orbital raising and lowering operators indicate that they are "normalized" by the effective value of $l(l+1)$ for the molecule. A parity conserving basis including all relevant angular momenta is constructed using functions appropriate to the unquenched limit. Rotational energy levels are calculated via diagonalization of the complete Hamiltonian matrix that results within the framework of the two interacting electronic states, ${ }^{2} A^{\prime}$ and ${ }^{2} A^{\prime \prime}$.

In both limiting cases, that of negligible energy difference between the ${ }^{2} A^{\prime}$ and ${ }^{2} A^{\prime \prime}$ states as well as that of wellseparated states, the rotational energy levels group into manifolds according to the value for the projection of an angular 
momentum along the $a$ inertial axis of the complex. Figure 3 shows the energies of these manifolds, referenced to a common energy origin, as a function of the ratio of the difference potential to the monomer spin-orbit coupling constant $a$. The logarithmic scale, $\ln \sqrt{\left(\rho^{2} / a^{2}+1\right)}$, chosen for the abscissa of the figure reflects the strength of the coupling responsible for the quenching of orbital angular momentum. It also allows for easy visualization of the strong dependence of the energies on the difference potential for small values of the ratio while compressing the weaker dependence at larger ratios. On the left side of the figure, with $\rho=0$, the manifolds are characterized by the half integer projection $P$ of the total angular momentum $J$ with contributions from molecular rotation, electronic orbital, and electronic spin angular momentum. For each level shown in this lower of the two spin-orbit components, the projections of the electronic orbital and spin angular momenta sum to give $|\lambda+\sigma|=|\omega|=3 / 2$. The superscript indicates the sign of $\omega$. As the difference potential increases, the electronic orbital angular momentum is quenched and with it the magnetic field responsible for coupling the electron spin to the $a$ inertial axis. Eventually, when the difference potential is much larger than the spin-orbit splitting, the electronic orbital angular momentum is fully quenched, and the limit on the right side of Fig. 3 is reached. Now, the appropriate angular momentum $N$ is an integer as it excludes electron spin, and the manifolds are labeled with the projection of $N, K_{a}$. Since the acetylenic $\mathrm{CH}$ stretching vibration leads to a $b$-type infrared band in the complex, the projection quantum number changes in the transition from the ground to excited vibrational state. Consequently, the transition frequencies, and in turn, the band contour will be sensitive functions of the difference potential.

The changes in the band contour with the magnitude of the difference potential are clearly seen in Fig. 2. Infrared spectra for several values of the difference potential are simulated using intensities calculated by transforming the electric dipole transition moment matrix into the energy eigenvector basis and including the appropriate Boltzmann and nuclear spin statistical weight factors. ${ }^{5}$ The linewidth and rotational temperature chosen for the figure match the experimental values as discussed below. The bottom trace shows the contour expected for completely unquenched electronic orbital angular momentum, i.e., $\rho=0$. The predominant features in this spectrum are $Q$-branches originating from the lowest three manifolds on the left side of Fig. 3. The right subscript on the branch label indicates the value of $P^{\prime \prime}$, and the left superscript the value of $\Delta P$ in a straightforward extension of symmetric top branch notation. With the exception of the ${ }^{p} Q_{1 / 2}$ branch, which has $P^{\omega^{\prime \prime}} \rightarrow P^{\omega^{\prime}}=1 / 2^{+}$ $\rightarrow 1 / 2^{-}$, the sign of $\omega$ does not change. Traces for larger magnitudes of the difference potential are shown as one moves up in Fig. 2. Significant changes occur until the electronic orbital angular momentum is completely quenched, and the contour takes the limiting form at the top. $Q$ branches again dominate the spectrum and are labeled with the usual ${ }^{\Delta K_{a}} \Delta N_{K_{a}^{\prime \prime}}$ notation of near prolate asymmetric tops. For intermediate magnitudes of the difference potential, the characterization of the predominant features is less obvious as $N$ and $K_{a}$ replace $J$ and $P$ as the better labels. Neverthe-
TABLE I. Spectroscopic parameters $\left(\right.$ in $\mathrm{cm}^{-1}$ ) determined from a fit to the band contour of the $\mathrm{CH}$ stretching fundamental of $\mathrm{OH}$-acetylene. [Values in parentheses are $2 \sigma$ uncertainties in the last digit.]

\begin{tabular}{lc}
\hline \hline$\nu_{0}$ & $3278.64(1)$ \\
$A^{\prime \prime}$ & $1.217(8)$ \\
$A^{\prime}$ & $1.202(4)$ \\
$(B+C) / 2^{\mathrm{a}}$ & $0.1399^{\mathrm{b}}$ \\
$(B-C) / 2^{\mathrm{a}}$ & $0.01^{\mathrm{c}}$ \\
$\rho^{\mathrm{a}}$ & $-148.1(7)$ \\
\hline
\end{tabular}

${ }^{a}$ Common value for both upper and lower vibrational state.

${ }^{b}$ Fixed at Coriolis-corrected value from Ref. 4.

${ }^{c}$ Fixed at the value calculated using the geometry determined in Ref. 4.

less, it is possible to follow the features as the spectrum moves from one limit to the other. The black (gray) bars in Fig. 2 show how the ${ }^{p} Q_{3 / 2}\left({ }^{r} Q_{3 / 2}\right)$ feature evolves into the ${ }^{p} Q_{1}\left({ }^{r} Q_{1}\right)$ as $|\rho|$ increases.

The simulation in Fig. 2 for $\rho=-148 \mathrm{~cm}^{-1}$ is overlaid with the experimental spectrum and is the result of a least squares fit to determine the difference potential as well as several other model parameters for the $\mathrm{OH}$-acetylene complex. This best fit value for $\rho$ corresponds to $\ln \sqrt{\left(\rho^{2} / a^{2}+1\right)}=0.759$ on the abscissa of the correlation diagram shown in Fig. 3. Since individual rotational lines are not resolved in the experimental spectrum, the rotational constant $(B+C) / 2$ is fixed at the Coriolis corrected value $\left(0.1399 \mathrm{~cm}^{-1}\right)$ determined from the $\mathrm{OH}$ overtone spectrum of the complex, ${ }^{4}$ and $(B-C) / 2=0.01 \mathrm{~cm}^{-1}$ is calculated from the molecular geometry. Both constants were assumed to remain unchanged upon vibrational excitation, as the simulated spectrum was insensitive to reasonable values for such changes. Preliminary fits indicated that the rotational temperature of the complex in the molecular jet for this experiment, $T_{\text {rot }}$, is $\approx 6 \mathrm{~K}$ and that the experimental linewidth $\Gamma$ is $0.30 \mathrm{~cm}^{-1}$ (Lorentzian). Fits in which these parameters were allowed to float were not very stable, however, so they were fixed to these values in the final fit. The values of those parameters allowed to float (vide infra) did not depend on the precise values chosen for $T_{\text {rot }}$ and $\Gamma$. Ultimately, in addition to the difference potential, the band origin, the $A$ rotational constant in the ground vibrational state and its change upon vibrational excitation, were determined in the fit to the observed band contour and are presented in Table I. Although an overall scaling factor (not reported) was also allowed to vary in the fit, it is important to note that the relative intensities of the features in the simulated band are fixed by the electric dipole transition moments calculated by the model and the rotational temperature of the complex in the jet taking into account the effects of nuclear spin statistics. The band contour does not allow a determination of the sign for the difference potential, but $a b$ initio calculations ${ }^{4}$ indicate that the ${ }^{2} A^{\prime \prime}$ electronic state lies below the ${ }^{2} A^{\prime}$ in the complex, so that the reported value is negative. The $0.015 \mathrm{~cm}^{-1}$ difference between the $A$ rotational constants for the two vibrational states is determined only by the weak feature just below $3274 \mathrm{~cm}^{-1}$ in the spectrum. Fits with $\Delta A$ constrained to zero failed to reproduce the position of this feature, although the rest of the band continued to match well. In spite of the agreement so obtained, it is not clear how much sig- 
nificance should be attached to this determination for $\Delta A$ given the low signal to noise ratio for the peak.

The agreement between the observed spectrum and that simulated using the results from the fit is remarkably good. The positions, shapes, and relative intensities of all the major features and several minor ones are all reproduced. The worst agreement occurs at 3276.5 and $3283.1 \mathrm{~cm}^{-1}$ where the simulated spectrum has somewhat greater intensity than that observed. Interestingly, the transitions, ${ }^{p} P(5 / 2)_{5 / 2}$ and ${ }^{r} R(5 / 2)_{5 / 2}$, responsible for the majority of the intensity at these two IR wave numbers are from the same initial rotational level, namely, the two nearly degenerate parity components of $(J, P, \omega)=(5 / 2,5 / 2,+3 / 2)$. Dividing the predicted intensity for these lines by a factor of 2 brings the simulation into near perfect agreement with experiment at these points without noticeably changing the simulation at other wavenumbers. The otherwise excellent agreement between model and experiment for the vast majority of the band suggests that the disagreement arises from some effect specific to this initial level. Within the context of the two interacting electronic states included in the model, however, there is no obvious candidate for a perturbing interaction that would lead to this discrepancy.

The linewidth required to simulate the experimental spectrum, $\Gamma=0.30 \mathrm{~cm}^{-1}$ (Lorentzian), is significantly broader than the IR laser bandwidth $\left(0.15 \mathrm{~cm}^{-1}\right.$, Gaussian). The excess linewidth is not likely, however, to be a result of lifetime broadening in the predissociating excited vibrational state of $\mathrm{OH}$-acetylene. The $\mathrm{OH}$ overtone band of the complex showed no broadening beyond the same laser bandwidth after excitation of a vibration more directly coupled to the dissociation at over twice the energy than the $\mathrm{CH}$ fundamental, ${ }^{4}$ and the lifetime is expected to be longer for this $\mathrm{CH}$ stretch band. In the analogous complexes of acetylene with HF (Refs. 10 and 20) and HCN (Ref. 14) the lifetimes of the acetylene asymmetric $\mathrm{CH}$ stretch states are significantly longer than those obtained by excitation of the fundamentals of the $\mathrm{HX}$ stretch. The case is less clear cut for $\mathrm{HCl}$-acetylene ${ }^{11}$ where lines in the asymmetric $\mathrm{CH}$ stretching fundamental band show $J$-dependent linewidths of $0.007-$ $0.033 \mathrm{~cm}^{-1}$, somewhat broader than the $0.006 \mathrm{~cm}^{-1}$ observed after excitation of the $\mathrm{HCl}$ stretch. Nevertheless, these are all much narrower than the $0.3 \mathrm{~cm}^{-1}$ value seen in this band of $\mathrm{OH}$-acetylene. Narrower linewidths are also seen in the spectra of rare gas-acetylene complexes where the unquenched Fermi resonance between $\nu_{3}$ and $\nu_{2}+\nu_{4}+\nu_{5}$ in the acetylene monomer enhances the rate of vibrational predissociation. ${ }^{17,21}$ Even though the coupling that remains after complexation breaks the resonance has been suggested as a key influence in determining the final state of the departing acetylene fragment in the predissociation of $\mathrm{HF}-$ acetylene ${ }^{22}$ and $\mathrm{HCl}$-acetylene, ${ }^{23}$ there is nothing to suggest that the coupling is enhanced in $\mathrm{OH}$-acetylene leading to rapid redistribution of energy following $\mathrm{CH}$ stretching excitation. We believe that the linewidth in the observed spectrum is most likely a consequence of IR power broadening. Unfortunately, low signal levels in this experiment prevented us from determining the effect of reduced laser power on the linewidth.

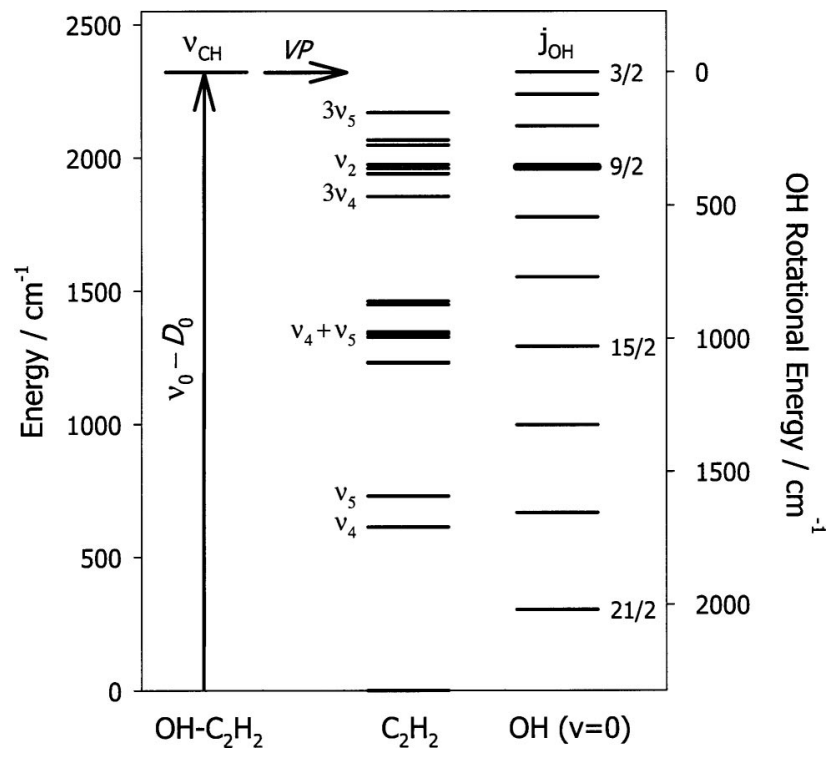

FIG. 4. Energy level diagram illustrating the vibrational predissociation (VP) dynamics of $\mathrm{OH}$-acetylene reactant complexes prepared with acetylenic $\mathrm{CH}$ stretch excitation $\left(\nu_{\mathrm{CH}}\right)$. The arrow on the left-hand side indicates the total energy available to the fragments, $\nu_{0}-D_{0}$, with the zero of energy defined as the $\mathrm{OH} X^{2} \Pi_{3 / 2}(v=0, j=3 / 2)+\mathrm{C}_{2} \mathrm{H}_{2}$ asymptote. The central region shows the energetically allowed vibrational states of the inelastically scattered $\mathrm{C}_{2} \mathrm{H}_{2}$ fragments. The right hand column and axis illustrate the allowed rotational levels $\left(j_{\mathrm{OH}}\right)$ of the $\mathrm{OH} X^{2} \Pi_{3 / 2}(v=0)$ fragments and associated rotational energy. The most populated $\mathrm{OH}$ product state, $j_{\mathrm{OH}}$ $=9 / 2$ (highlighted), is consistent with intramolecular energy transfer to the $\nu_{2} \mathrm{C} \equiv \mathrm{C}$ stretch of acetylene.

Similarly, detailed measurements of the quantum state distribution of the $\mathrm{OH}$ fragments resulting from vibrational predissociation of the $\mathrm{OH}$-acetylene $\left(\nu_{\mathrm{CH}}\right)$ complex were not made due to small signal levels and large background levels for the lowest $\mathrm{OH}$ rotational states. Nevertheless, it was possible to determine that the $\mathrm{OH} X^{2} \Pi_{3 / 2}\left(v=0, j_{\mathrm{OH}}\right)$ fragments contain a relatively low amount of rotational excitation, spanning primarily from $j_{\mathrm{OH}}=5 / 2$ to $11 / 2$. For the most populated product state, $j_{\mathrm{OH}}=9 / 2$ with only $355 \mathrm{~cm}^{-1}$ of rotational excitation, and using the previously determined upper limit for the dissociation energy of $\mathrm{OH}$-acetylene, $D_{0}$ $\leqslant 956 \mathrm{~cm}^{-1},{ }^{4}$ there remains over $1967 \mathrm{~cm}^{-1}$ of energy ${ }^{24}$ to be accommodated in the acetylene fragment and/or through translational recoil after excitation of one quantum of asymmetric $\mathrm{CH}$ stretch in the complex. Predissociation following excitation of this mode in the analogous closed shell complexes $\mathrm{HF}$-acetylene ${ }^{22}$ and $\mathrm{HCl}$-acetylene ${ }^{23}$ proceeds via an intramolecular vibration to vibration $(V-V)$ energy transfer process to the $\nu_{2} \mathrm{C} \equiv \mathrm{C}$ stretching mode of acetylene with low rotational excitation of the acetylene fragment and little translational recoil, as expected based on energy gap arguments. ${ }^{25}$ A similar mechanism is expected for OHacetylene. Indeed, Fig. 4 shows that excitation of $\nu_{2}$ in the departing acetylene fragment corresponds nicely to the observed peak in the $\mathrm{OH}$ fragment rotational distribution at $j_{\mathrm{OH}}=9 / 2$. The $2 \nu_{4}+\nu_{5}$ acetylene bending vibrational state is nearly degenerate with the $\mathrm{C} \equiv \mathrm{C}$ stretching state, but was definitively eliminated as an exit channel in the vibrational predissociation of HF-acetylene. ${ }^{22}$ Dissociation into this state and the other nearby states involving three quanta distributed 
amongst the $\nu_{4}$ and $\nu_{5}$ bending vibrations of acetylene is unlikely because of the large number of vibrational quantum numbers that would have to change. ${ }^{26}$ Additionally, the apparent lack of dissociation into channels with greater amounts of rotational excitation $\left(>500 \mathrm{~cm}^{-1}\right)$ indicates that excitation of one or two quanta of acetylene bending vibrations also plays a minor role, at best, in the predissociation dynamics of $\mathrm{OH}$-acetylene.

\section{DISCUSSION AND CONCLUSIONS}

The $b$-type, $\mathrm{CH}$ stretching band of the $\mathrm{OH}$-acetylene reactant complex clearly shows the effects of partial quenching of $\mathrm{OH}$ orbital angular momentum upon complex formation as predicted by earlier model calculations. ${ }^{5}$ The value of the energy difference between the ${ }^{2} A^{\prime}$ and ${ }^{2} A^{\prime \prime}$ electronic states, or difference potential, is determined to be $-148.1 \pm 0.7$ $\mathrm{cm}^{-1}$, remarkably close to the ab initio prediction of -140 $\mathrm{cm}^{-1},{ }^{4}$ given that the latter value is determined from the calculated energies for the two states at their respective equilibrium geometries and that the experimental value reflects the effects of averaging over the vibrational zero-point motion of the complex. This determination allows us to address an unresolved issue in the $\mathrm{OH}$ overtone spectrum. In that band of the complex, the sole observable effect of the difference potential is on the breadth of the features in the $P$ and $R$ branches. The present analysis confirms that the excess linewidth $\left(0.19 \mathrm{~cm}^{-1}\right)$ observed in the $\mathrm{OH}$ overtone spectrum ${ }^{4}$ results from inhomogeneous broadening due to the partial quenching of orbital angular momentum.

The degree of quenching corresponding to this difference potential depends on the rotational and spin-orbit state of the complex, but for the low $P, \omega=+3 / 2$ levels that contribute to the observed spectrum under jet conditions, the complex has $\approx 85 \% A^{\prime \prime}$ character and $15 \% A^{\prime} .{ }^{27}$ At this level of mixing, $\approx 2 / 3$ of the orbital angular momentum remains unquenched as measured by the expectation value of the effective spin-orbit interaction operator $l_{z} s_{z}$. The rotationally resolved spectrum of the $\mathrm{OH}$ overtone band of the complex, ${ }^{4}$ shows that this is still sufficient orbital angular momentum to keep the electron spin coupled to the inertial axes of the molecule. The contour of the $\mathrm{CH}$ stretching band, however, demonstrates that this amount of quenching has dramatic effects on the rotational motion of the complex.

The results obtained here for the $\mathrm{CH}$ stretching band of $\mathrm{OH}$-acetylene confirm and support the structural comparisons made to analogous closed-shell, $\pi$-type hydrogen bonded complexes of acetylene in our previous paper. ${ }^{4}$ Even closer similarities in spectroscopic properties are seen here, since the vibrational excitation is localized on the acetylene moiety. The $-9.75 \mathrm{~cm}^{-1}$ shift in vibrational band origin for the $\mathrm{CH}$ stretching vibration in $\mathrm{OH}$-acetylene is remarkably similar to those observed for the $\mathrm{HF}, \mathrm{HCl}$, and $\mathrm{HCN}$ complexes and listed in Table II. The $A^{\prime \prime}$ rotational constant in each case is slightly larger than the rotational constant $(B$ $=1.176642 \mathrm{~cm}^{-1}$ ) (Ref. 28) of the acetylene monomer, as expected from vibrational averaging about the $T$-shaped equilibrium geometry. The change in the $A$ rotational constant upon excitation of the asymmetric $\mathrm{CH}$ stretching vibration is likewise similar to the analogous change in the mono-
TABLE II. Vibrational band origin shift and change in $A$ rotational constants for the asymmetric $\mathrm{CH}$ stretching fundamental in $\mathrm{XH}$-acetylene complexes.

\begin{tabular}{lccc}
\hline \hline $\begin{array}{c}\text { Complex } \\
\mathrm{HCCH}-\end{array}$ & $\Delta \nu_{0}{ }^{\mathrm{a}} / \mathrm{cm}^{-1}$ & $A^{\prime \prime} / \mathrm{cm}^{-1}$ & $\Delta A / \mathrm{cm}^{-1}$ \\
\hline $\mathrm{OH}^{\mathrm{b}}$ & $-9.75(1)$ & $1.217(8)$ & $-0.015(6)$ \\
$\mathrm{HF}^{\mathrm{c}}$ & -12.102 & 1.19260 & -0.00570 \\
$\mathrm{HCl}^{\mathrm{d}}$ & -10.313 & 1.20363 & -0.00960 \\
$\mathrm{HCN}^{\mathrm{e}}$ & -10.853 & 1.20102 & -0.00549 \\
\hline \hline
\end{tabular}

${ }^{\text {a Calculated relative to the deperturbed } \nu_{3} \text { band origin, } 3288.391 \mathrm{~cm}^{-1} \text {, of }}$ acetylene. Reference 28.

${ }^{\mathrm{b}}$ This work, values in parenthesis are $2 \sigma$ uncertainties in the last digit.

${ }^{\mathrm{c}}$ Reference 10 .

${ }^{\mathrm{d}}$ Reference 11 .

${ }^{\mathrm{e}}$ Reference 14.

mer, $\Delta B_{\mathrm{HCCH}}=-0.00422 \mathrm{~cm}^{-1}$. The value of $\Delta A$ for $\mathrm{OH}-$ acetylene is somewhat larger than the related closed-shell complexes, but as noted earlier, this quantity may not be well determined in the fit.

The strong structural correspondences and the striking similarities in predissociation dynamics discussed in the previous section would appear to suggest that the equilibrium properties and inelastic scattering of $\mathrm{OH}$-acetylene are determined by the same long range interactions as those responsible for the closed-shell analogs, such as HF-acetylene. That is, the potential energy surface in the vicinity of the T-shaped reactant complex results primarily from electrostatic interactions of both permanent and induced multipole moments as well as quantum mechanical dispersion forces. While true, this simple view neglects an important consequence of complexation on the $\mathrm{OH}$ monomer. Namely, the spectroscopy of the $\mathrm{OH}$-acetylene complex provides evidence that the changes in the electronic description of the molecule necessary for the evolution of reactants into product have already begun to occur when the centers of mass of the reactive species are $3.327 \AA$ apart on average. The long range forces responsible for complex formation and often assumed to have negligible effect on the electronic environment of the monomers cause a partial quenching of $\mathrm{OH}$ electronic orbital angular momentum in $\mathrm{OH}$-acetylene and lift the associated orbital degeneracy. This may be seen as the start of the process of reaction that ultimately leads to complete quenching and well-separated, orbitally nondegenerate states characteristic of the addition product, $\mathrm{HCCHOH}$.

The continuing changes in the nature of the electronic states of the system as the $\mathrm{OH}$ fine structure $\left({ }^{2} \Pi_{i}\right)$ splitting evolves into the ${ }^{2} A^{\prime}$ and ${ }^{2} A^{\prime \prime}$ states of $\mathrm{HCCHOH}$ will have a profound effect on the height, shape and location of energy barriers, ${ }^{29}$ and it will be important to consider that the nuclear motion leading to reaction occurs on these two coupled states. Closer to the transition state, covalent interactions will start to play an important role in the nature of the ${ }^{2} A^{\prime}$ surface, and it would be extremely valuable to have a theoretical characterization of the minimum energy path from the region of the attractive well in the entrance channel to the transition state. Although significant departures from the $T$-shaped geometry and monomer bond lengths of the reactant complex will occur along this path, the $\mathrm{OH}$ - 
acetylene complex provides a snapshot at the beginning of the journey towards reaction.

\section{ACKNOWLEDGMENTS}

This research was sponsored by the Office of Basic Energy Sciences of the Department of Energy. M.D.M. thanks the H. Axel Schupf' 57 Fund for Intellectual Life at Amherst College for sabbatical leave support.

${ }^{1}$ B. J. Finlayson-Pitts and J. N. Pitts, Jr., Chemistry of the Upper and Lower Atmosphere (Academic, San Diego, 2000).

${ }^{2}$ I. Glassman, Combustion (Academic, San Diego, 1996).

${ }^{3}$ M. I. Lester, B. V. Pond, M. D. Marshall, D. T. Anderson, L. B. Harding, and A. F. Wagner, Faraday Discuss. 118, 373 (2001).

${ }^{4}$ J. B. Davey, M. E. Greenslade, M. D. Marshall, M. I. Lester, and M. D. Wheeler, J. Chem. Phys. 121, 3009 (2004).

${ }^{5}$ M. D. Marshall and M. I. Lester, J. Chem. Phys. 121, 3019 (2004).

${ }^{6}$ M. Tsiouris, M. D. Wheeler, and M. I. Lester, J. Chem. Phys. 114, 187 (2001).

${ }^{7}$ L. S. Rothman, C. P. Rinsland, A. Goldman et al., J. Quant. Spectrosc. Radiat. Transf. 60, 665 (1998).

${ }^{8}$ G. H. Dieke and H. M. Crosswhite, J. Quant. Spectrosc. Radiat. Transf. 2, 97 (1962).

${ }^{9}$ M. T. Berry, M. R. Brustein, and M. I. Lester, J. Chem. Phys. 92, 6469 (1990).

${ }^{10}$ Z. S. Huang and R. E. Miller, J. Chem. Phys. 90, 1478 (1989).

${ }^{11}$ D. C. Dayton, P. A. Block, and R. E. Miller, J. Phys. Chem. 95, 2881 (1991).
${ }^{12}$ G. T. Fraser, R. D. Suenram, F. J. Lovas, A. S. Pine, J. T. Hougen, W. J. Lafferty, and J. S. Muenter, J. Chem. Phys. 89, 6028 (1988).

${ }^{13}$ D. G. Prichard, R. N. Nandi, and J. S. Muenter, J. Chem. Phys. 89, 115 (1988).

${ }^{14}$ P. A. Block, K. W. Jucks, L. G. Pedersen, and R. E. Miller, Chem. Phys. 139, 15 (1989).

${ }^{15}$ T. A. Hu, D. G. Prichard, L. H. Sun, J. S. Muenter, and B. J. Howard, J. Mol. Spectrosc. 153, 486 (1992).

${ }^{16}$ R. J. Bemish, P. A. Block, L. G. Pedersen, W. T. Yang, and R. E. Miller, J. Chem. Phys. 99, 8585 (1993).

${ }^{17}$ R. J. Bemish, L. Oudejans, R. E. Miller, R. Moszynski, T. G. Heijmen, T. Korona, P. E. Wormer, and A. van der Avoird, J. Chem. Phys. 109, 8968 (1998).

${ }^{18}$ Z. S. Huang and R. E. Miller, Chem. Phys. 132, 185 (1989).

${ }^{19}$ D. K. Russell and H. E. Radford, J. Chem. Phys. 72, 2750 (1980).

${ }^{20}$ Z. S. Huang and R. E. Miller, J. Chem. Phys. 86, 6059 (1987).

${ }^{21}$ R. J. Bemish and R. E. Miller, Chem. Phys. Lett. 281, 272 (1997).

${ }^{22}$ L. Oudejans, D. T. Moore, and R. E. Miller, J. Phys. Chem. 110, 209 (1999).

${ }^{23}$ L. Oudejans and R. E. Miller, J. Phys. Chem. A 103, 4791 (1999).

${ }^{24}$ The available energy increases if $D_{0}$ is less than its upper limit and from the small rotational energy of the $\mathrm{OH}$-acetylene complex at $6 \mathrm{~K}$.

${ }^{25}$ J. A. Beswick and J. Jortner, Adv. Chem. Phys. 47, 363 (1981).

${ }^{26}$ G. E. Ewing, J. Phys. Chem. 91, 4662 (1987).

${ }^{27}$ This assumes the $a b$ initio sign for $\rho$. Reversing the sign would swap the amounts of ${ }^{2} A^{\prime}$ and ${ }^{2} A^{\prime \prime}$ character.

${ }^{28}$ B. C. Smith and J. S. Winn, J. Chem. Phys. 89, 4638 (1988).

${ }^{29}$ M. P. Deskevich, D. J. Nesbitt, and H. J. Werner, J. Chem. Phys. 120, 7281 (2004). 